\title{
A Case of Subcutaneous Implantable Cardioverter-Defibrillator Twiddler's Syndrome
}

\author{
Chengyue Jin ${ }^{1}$, Daniel Frenkel ${ }^{1}$, Rhadamas Rojas ${ }^{1}$, Jason Jacobson ${ }^{1}$, Sei Iwai $^{1}$, and Aileen \\ M. Ferrick ${ }^{1}$
}

${ }^{1}$ Westchester Medical Center

October 8, 2020

\begin{abstract}
Twiddler's syndrome is a rare complication where a pacemaker or implantable cardioverter-defibrillator (ICD) is displaced with or without patient manipulation of their device. There are reports on transvenous devices but a paucity of data on subcutaneous devices. A 50-year-old male with hypertrophic cardiomyopathy and non-sustained ventricular tachycardia underwent subcutaneous-ICD (S-ICD) implantation for primary prevention. Remote device interrogation 4 weeks after device placement reported a shock due to "ventricular fibrillation". It also showed abnormal lead impedance. Chest X-ray showed lead was dislodged and coiled around the pulse generator. Patient underwent lead revision and device replacement without further complications.
\end{abstract}

\section{Introduction}

Twiddler's syndrome refers to deliberate or unintentional twisting of the pulse generator in a device pocket resulting in lead displacement. This will usually present as a malfunction of the pacemaker or ICD. ${ }^{1}$ Patients may be asymptomatic or can present with a variety of symptoms including inappropriate shocks, failure to capture, diaphragmatic contraction, pectoral muscle stimulation, or brachial plexus stimulation. ${ }^{2}$ In the past 50 years, twiddler's syndrome with transvenous ICDs (TV-ICD) and pacemakers has been well-described and thoroughly studied. However, there are limited data on twiddler's syndrome with the S-ICDs. We report a case of S-ICD twiddler's syndrome and a review of the current perspective of twiddler's syndrome.

\section{Case presentation}

The patient is a 50-year-old male with a past medical history of hypertrophic cardiomyopathy, non-sustained ventricular tachycardia, and evidence of late gadolinium enhancement on MRI consistent with fibrosis. After a shared decision-making discussion, he underwent implantation of S-ICD for primary prevention of sudden cardiac death. Of note, the pulse generator was secured to the fascia with two 0-Silk sutures as part of standard procedure and two non-absorbable, braided sutures were placed at the sub-xiphoid incision to tie down the lead around the suture sleeve, and the lead was tunneled up along the sternum. During the first follow-up visit 2 weeks post-implant, the S-ICD interrogation showed appropriate device function. Two weeks later, remote monitoring reported one ICD shock due to an event categorized as ventricular fibrillation (Figure 1). Interrogation demonstrated reduced $\mathrm{R}$ wave amplitude and an abnormal impedance of $7 \mathrm{ohms}$ during shock delivery. Patient reported no symptoms prior to the shock but was clapping hands to music when it occurred. A chest X-ray revealed the S-ICD lead was dislodged and retracted, with coiling around the generator in left lateral chest wall (Figure 2A and 2B) compared to the original post-implant position (Figure 2C and 2D). Subsequently, the patient underwent lead revision and device replacement. To reduce the risk of future dislodgement, during re-implantation, an incision was made near the expected position of the tip of the ICD lead, which was then secured with 0-Silk to prevent dislodgment. 


\section{Discussion}

Twiddler's syndrome is a rare complication of cardiac implantable electronic devices resulting in device malfunction due to lead migration and dislodgement that was first described by Bayliss in $1968^{1}$ Other variants of "idiopathic lead migration syndrome" 3 have been described including "reel syndrome" (in which the lead is retracted along the generator, similar to a fishing reel), and "ratchet syndrome" (where the suture sleeve acts as a unidirectional "brake")." The incidence of twiddler's syndrome reported in the literature range from $0.07 \%$ to $2.69 \% .{ }^{4,5}$ The single most important risk factor for twiddler's syndrome is manual manipulation of the device by the patient ${ }^{3}$ although "iatrogenic" twiddler's syndrome has also been reported ${ }^{6}$. Advanced and younger ages, redundant skin, obesity, oversized pocket, and a history of a psychiatric disorder (especially obsessive-compulsive disorder) are considered other possible risk factors. 2,3,7 A left pectoral implant and silicone-insulated electrodes are also more commonly seen in patients developing twiddler's syndrome. ${ }^{3}$

Although many cases of twiddler's syndrome with transvenous pacemakers or ICDs have been reported, S-ICD-related lead migration has only previously been reported in one pediatric case. ${ }^{8}$ Compared to TVICD, the S-ICD has been shown to have a lower rate of early lead displacement within 30 days of device implantation (1\% vs $2.7 \%$ ), and lead malfunction (0\% vs. $6.2 \%) .{ }^{9}$ Additionally, complication rates of SICD implants rapidly decline after the proceduralist's first four implants and stabilize after 13 implant procedures. $^{10}$

Device malfunction as a result of twiddler's syndrome can be potentially fatal due to undetected or unsuccessful therapy for ventricular tachyarrhythmia. Routine workup for suspected cases of twiddler's syndrome includes electrocardiogram, posterior-anterior and lateral chest X-ray, as well as device interrogation. ${ }^{3}$ Replacement of a new lead and repositioning of pulse generator are often required to fully restore device function.

Studies have shown that certain surgical techniques can reduce the risk of twiddler's syndrome in TVICD placement, such as anchoring sutures to pectoralis fascia, antimicrobial pouches, and woven Dacron pouches. ${ }^{11-13}$ It is also reasonable to implant the device sub-muscularly in patients with a high risk of loose subcutaneous tissue, especially patients of advanced age, and those who are obese or female. Patient education can be helpful but not completely effective, given manipulation of the device may be subconscious and in some cases device migration may be spontaneous. ${ }^{3}$ Considering that the mechanisms for twiddler's syndrome in TV-ICD can also be relevant for S-ICD leads, it is reasonable to infer that the aforementioned strategies would also reduce the risk of S-ICD twiddler's syndrome.

\section{Conclusion}

Twiddler's syndrome can be a potentially dangerous, and even fatal, complication after ICD implantation, not only with TV-ICDs but also involving S-ICDs. Therefore, it is of paramount importance to diagnose and intervene early to avoid morbidity and mortality in these patients.

\section{Key Teaching Points}

- Twiddler's syndrome refers to the deliberate or unintentional twisting of the pulse generator in a device pocket resulting in lead displacement.

- It is of paramount importance to diagnose and intervene early to avoid morbidity and mortality in patients with both transvenous and subcutaneous ICDs.

- Certain surgical techniques can reduce the risk of twiddler's syndrome in TV-ICD placement, such as anchoring sutures to pectoralis fascia, non-absorbable antimicrobial pouches, and woven Dacron pouches. These same strategies may be effective with S-ICDs.

\section{References:}

[1] Bayliss CE, Beanlands DS, Baird RJ: The pacemaker-twiddler's syndrome: a new complication of implantable transvenous pacemakers. Can Med Assoc J 1968; 99:371-373. 
[2] Arindam P, Achyut S, Imran A, Naveen Ganiga Sanjeeva C, Rabin C: Pacemaker Twiddler's syndrome: Review Through a Case Report. Journal of Cardiovascular Disease Research 2015; 6:148-151.

[3] Morales JL, Nava S, Marquez MF, Gonzalez J, Gomez-Flores J, Colin L, Martinez-Rios MA, Iturralde P: Idiopathic Lead Migration: Concept and Variants of an Uncommon Cause of Cardiac Implantable Electronic Device Dysfunction. JACC Clin Electrophysiol 2017; 3:1321-1329.

[4] Fahraeus T, Hoijer CJ: Early pacemaker twiddler syndrome. Europace : European pacing, arrhythmias, and cardiac electrophysiology : journal of the working groups on cardiac pacing, arrhythmias, and cardiac cellular electrophysiology of the European Society of Cardiology 2003; 5:279-281.

[5] Kwon CH, Choi JH, Kim J, Jo U, Lee JH, Lee WS, Kim YR, Lee SY, Whang KW, Yang J, Kim SH, Oh YS, Park KM, Nam GB, Choi KJ, Kim YH: Complications of Cardiac Perforation and Lead Dislodgement with an MRI-Conditional Pacing Lead: a Korean Multi-Center Experience. Journal of Korean medical science 2016; 31:1397-1402.

[6] Morin DP, Iwai S: Iatrogenic Twiddler's syndrome. J Interv Card Electrophysiol 2010; 29:135-137.

[7] Wang Y, Hou W, Zhou C, Yin Y, Lu S, Liu G, Duan C, Cao M, Li M, Toft ES, Zhang HJ: Metaanalysis of the incidence of lead dislodgement with conventional and leadless pacemaker systems. Pacing Clin Electrophysiol 2018; 41:1365-1371.

[8] Srivastava N, Kean A: Significant Lead Migration of a Subcutaneous Implantable CardioverterDefibrillator in a Pediatric Patient. Journal of Innovations in Cardiac Rhythm Management 2017; 8.

[9] Schinkel AF, Vriesendorp PA, Sijbrands EJ, Jordaens LJ, ten Cate FJ, Michels M: Outcome and complications after implantable cardioverter defibrillator therapy in hypertrophic cardiomyopathy: systematic review and meta-analysis. Circ Heart Fail 2012; 5:552-559.

[10] Knops RE, Brouwer TF, Barr CS, Theuns DA, Boersma L, Weiss R, Neuzil P, Scholten M, Lambiase PD, Leon AR, Hood M, Jones PW, Wold N, Grace AA, Olde Nordkamp LR, Burke MC: The learning curve associated with the introduction of the subcutaneous implantable defibrillator. Europace : European pacing, arrhythmias, and cardiac electrophysiology : journal of the working groups on cardiac pacing, arrhythmias, and cardiac cellular electrophysiology of the European Society of Cardiology 2016; 18:1010-1015.

[11] Osoro M, Lorson W, Hirsh JB, Mahlow WJ: Use of an antimicrobial pouch/envelope in the treatment of Twiddler's syndrome. Pacing Clin Electrophysiol 2018; 41:136-142.

[12] Sobstyl MR, Zabek M, Brzuszkiewicz-Kuzmicka G, Pasterski T: Dual Anchor Internal Pulse Generator Technique May Lower Risk of Twiddler's Syndrome: A Case Series and Literature Review. Neuromodulation 2017; 20:606-612.

[13] Shandling AH, Ellestad MH, Castellanet MJ, Messenger JC: Dacron-woven pacemaker pouch. Influence on long-term pacemaker mobility. Chest 1991; 99:660-662.

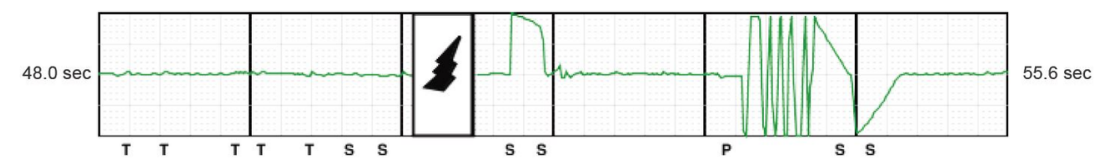

Figure 1. Electrogram showing inappropriate shock after device dislocation. 


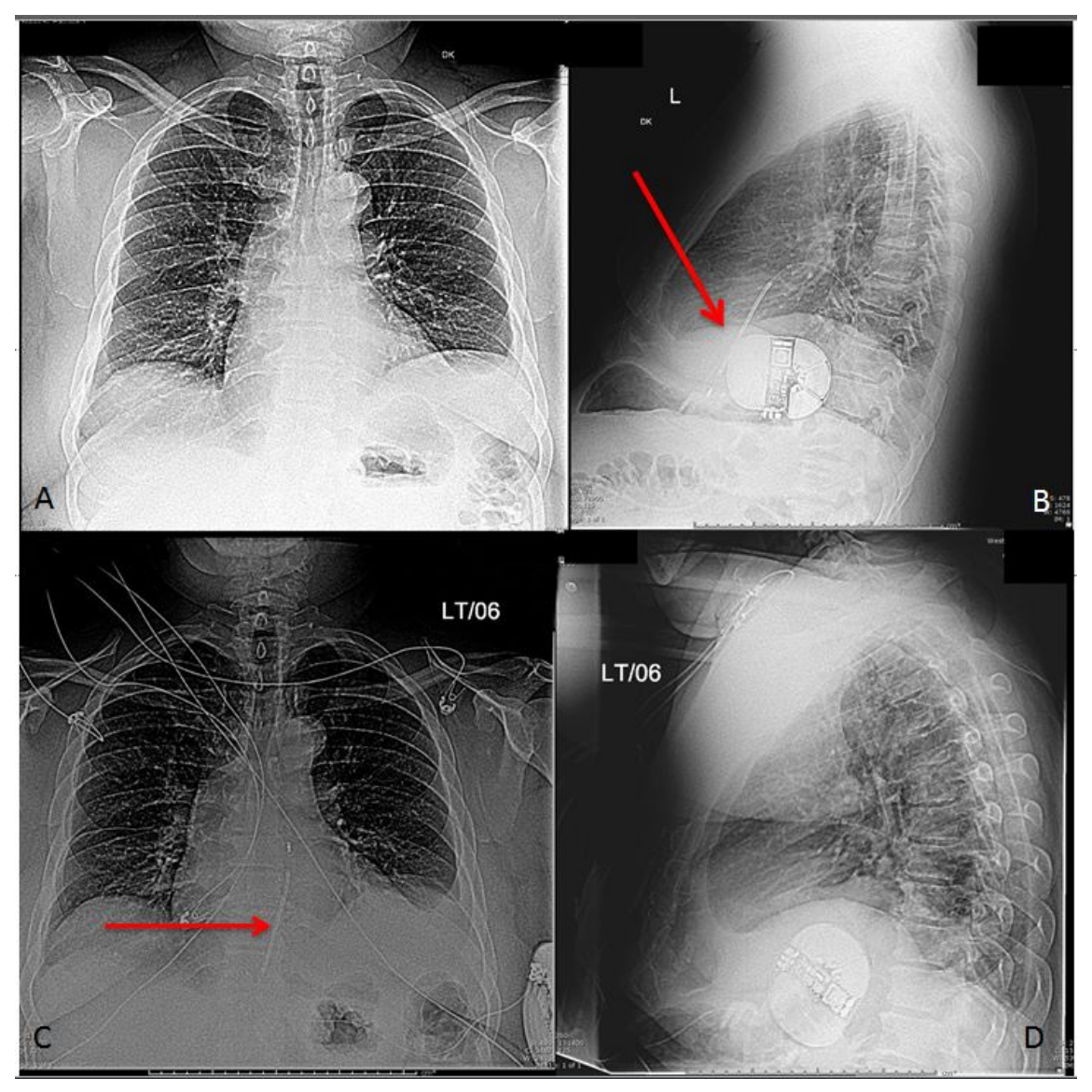

Figure 2. (A) and (B) Anterior-posterior and lateral Chest X-Rays showing subsequent lead dislodgement; (C) and (D) Anterior-posterior and lateral Chest X-Rays after initial implantation; Tip of lead indicated by red arrow. Note that the pulse generator is in a different orientation in the pocket compared to the original implant. 